\title{
Laser pulse duration dependence of blister formation on back- radiated Ti thin films for BB-LIFT
}

\author{
N. T. Goodfriend ${ }^{1} \cdot$ S. V. Starinskiy ${ }^{2} \cdot$ O. A. Nerushev $^{1} \cdot$ N. M. Bulgakova ${ }^{2,3}$ • \\ A. V. Bulgakov ${ }^{1,2}$ E. E. B. Campbell ${ }^{1,4}$
}

Received: 21 January 2016/ Accepted: 24 January 2016/Published online: 17 February 2016

(c) The Author(s) 2016. This article is published with open access at Springerlink.com

\begin{abstract}
The influence of the laser pulse duration on the mechanism of blister formation in the particle transfer technique, blister-based laser-induced forward transfer, was investigated. Pulses from a fs Ti:Sapphire laser (120 fs, $800 \mathrm{~nm}$ ) and from a ns Nd:YAG laser (7 ns, $532 \mathrm{~nm}$ ) were used to directly compare blister formation on thin titanium films of ca. $300 \mathrm{~nm}$ thickness, deposited on glass. The different blister morphologies were compared and contrasted by using optical microscopy and atomic force microscopy. The results provide evidence for different blister formation mechanisms: for fs pulses the mechanism is predominantly ablation at the metal-glass interface accompanied by confined plasma expansion and deformation of the remaining metal film; for ns pulses it is heating accompanied by thermal expansion of the metal film.
\end{abstract}

\section{Introduction}

The transfer of intact nanoparticles and large molecules from the solid to the gas phase is of considerable interest for mass spectrometry [1] and gas phase spectroscopy

E. E. B. Campbell

eleanor.campbell@ed.ac.uk

1 EaStCHEM and School of Chemistry, University of Edinburgh, David Brewster Road, Edinburgh EH9 3FJ, Scotland, UK

2 Kutateladze Institute of Thermophysics, Siberian Branch, Russian Academy of Sciences, Lavrentyev Ave. 1, Novosibirsk 630090, Russia

3 HiLASE Centre, Institute of Physics, ASCR, Za Radnicí 828, 25241 Dolní Břežany, Czech Republic

4 Division of Quantum Phases and Devices, School of Physics, Konkuk University, Seoul 143701, Korea techniques such as photoelectron spectroscopy [2] as well as for environmental monitoring applications [3] and more exotic studies related to fundamental quantum mechanics [4]. Multiple techniques have been developed to enable the gentle transfer, such as matrix-assisted laser desorption/ ionisation (MALDI), electrospray, and laser-induced acoustic desorption (LIAD) [5] being some of the most common. MALDI utilises the direct vaporisation of a matrix that contains the particles of interest. LIAD is similar to MALDI; however, the laser pulse impacts the back of a thin metal foil on which the molecules have been deposited. Electrospray involves spraying a solution of the particles of interest from a charged nozzle, where the charged droplets undergo evaporation and coulomb explosions until individual multiply charged particles remain. Whilst MALDI and electrospray methods are effective for producing beams of charged molecules and have revolutionised the mass spectrometry of large biomolecules, they harbour some significant drawbacks, such as difficulties in quantification, or the need to find an appropriate solution or matrix material that may also then be present in the produced beam, and neither of them are particularly suitable for preparing well-defined beams of neutral species. LIAD can be applied to any molecule that can be deposited on the metal foil and is thus more general; however, there is some controversy concerning the desorption mechanism and the extent to which molecules may be heated and fragment during the desorption process [6].

A technique known as blister-based-laser-induced forward transfer (BB-LIFT), closely related to LIAD, has been introduced and used mainly for depositing nanoparticles or organic molecules in well-defined microscale patterns on substrates [7-10]. More recently, it has been used to produce beams of nanoparticles for gas phase analysis [6]. This technique has the same advantages as LIAD but 
allows a more controlled desorption due to the control over the thickness and properties of the intermediate layer. A thin film of metal $[6,7,10]$ or polymer [8, 9] (used so far for depositing liquid inks) is deposited on a glass substrate and placed between the transparent substrate and the molecules of interest. The thin film absorbs the laser pulse thereby rapidly deforming into a blister that propels the particles or ink forward in a beam. The intermediate layer is not removed in this process but is mechanically deformed, thus imparting momentum to the desorbed particles. The technique has shown considerable promise for printing micro-scale patterns based on desorbed organic material [9, 11, 12] or nanoparticles [7]. However, there is some uncertainty concerning the method of blister formation and evidence for extensive heating of the metal film when using ns lasers [6], which could lead to thermal degradation of the desorbed nanoparticles and molecules. A comparison involving UV ns laser desorption of organic liquids using no intermediate layer, a thin metal layer and a thick polyimide layer did provide evidence of degradation in the first two cases but not with the thick polymer film [9].

There are two mechanisms that are mainly considered in the context of the BB-LIFT process, illustrated in Fig. 1. The first, usually discussed in the context of thin metal film layers [6], involves rapid heating accompanied by deformation induced by the different thermal expansion coefficients of the metal film and the underlying substrate. The second, mainly discussed in the context of ns UV laser BBLIFT of polyimide films [8], involves localised ablation at the interface between the polymer film and the underlying substrate. In this paper, we report the results of a study that provides a comparison of thin metal film deformation in BB-LIFT for laser pulse durations in the fs and ns regimes.
Evidence is found for a predominantly ablation mechanism for fs laser pulses and a thermal mechanism for ns pulses.

\section{Experimental details}

Thin Ti films with a thickness of ca. 200-400 nm were deposited onto glass microscope slides using electron beam evaporation under ultrahigh vacuum conditions. Titanium is an appropriate metal to use as the intermediate layer for BB-LIFT due to its reasonably high coefficient of thermal expansion $\left(9 \times 10^{-6} \mathrm{~K}^{-1}\right.$ for a 300-nm-thick film [13]), low thermal conductivity (ca. $20 \mathrm{Wm}^{-1} \mathrm{~K}^{-1}$ ), high melting point, $1941 \mathrm{~K}$ [14] and high tensile strength at high temperature [14]. The substrates were mounted on an $x$ $y$ translation stage and irradiated through the glass microscope slide under high vacuum conditions (base pressure of $3 \times 10^{-7}$ mbar). Two lasers were used for the irradiation: the second harmonic output of a Nd:YAG laser with $7 \mathrm{~ns}$ pulse duration at $532 \mathrm{~nm}$ (1/e beam diameter of $370 \pm 30 \mu \mathrm{m}$ at the metal film position, fluence range 220-370 $\mathrm{mJ} \mathrm{cm}^{-2}$ ) and the fundamental output from a Ti:Sapphire laser at $800 \mathrm{~nm}$ with $120 \mathrm{fs}$ pulse duration, (1/ $e$ beam diameter of $300 \pm 30 \mu \mathrm{m}$, fluence range $180-210 \mathrm{~mJ} \mathrm{~cm}^{-2}$ ), where the required fluence to induce BB-LIFT depends on film thickness, as shown previously by Kononenko et al. [7] for 50 ps duration 532-nm pulses. In both cases, the non-reflected laser energy was absorbed by the metal film. The reflection coefficients were measured to be 0.39 and 0.42 for 532 and $800 \mathrm{~nm}$, respectively, taking the glass substrate into account. The shot-to-shot fluctuation in the laser pulse energy was on the order of $5 \%$ (fs laser) and $15 \%$ (ns laser) giving error limits in the stated fluence values of around 20-25\%. The very different timescales of the laser pulses determine the rate of (a)

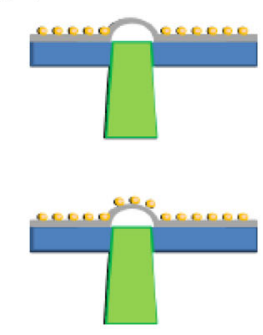

ponsenenesenen.

(b)

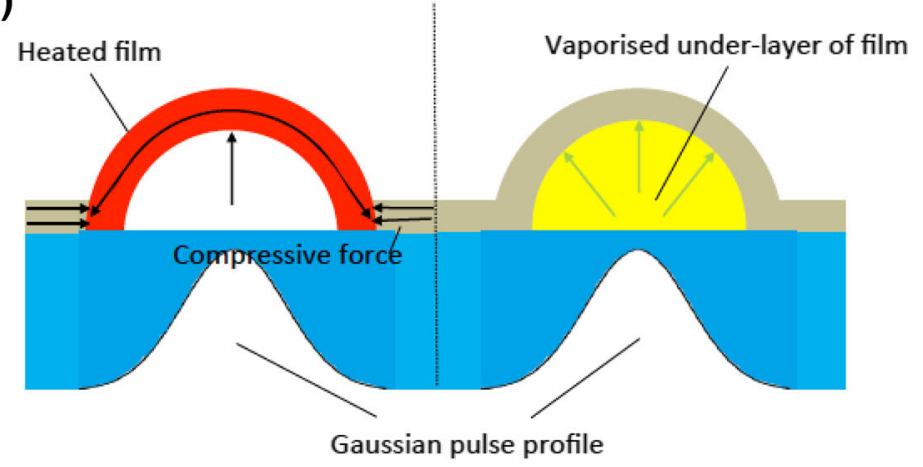

Fig. 1 a Schematic of the BB-LIFT process: the laser pulse (green) induces mechanical deformation in the thin film (grey) and removes the material deposited on the film (yellow dots). b Two possible mechanisms: Left heating of the thin film accompanied by

deformation due to thermal expansion. Right ablation at the interface between the thin film and the glass substrate leading to deformation due to expanding gas. Not to scale, dimensions in the vertical direction are greatly exaggerated for illustration purposes 
laser energy absorption and, correspondingly, the volume that is excited and the depth of the heat-affected zones in the metal film [15]. For the 800-nm fs pulse in Ti, the penetration depth of the laser pulse and heat-affected zone are much less than the film thickness. For ns pulses at $532 \mathrm{~nm}$, although the penetration depth is still much smaller than the film thickness, the film is heated almost uniformly across its thickness on the timescale of the laser pulse, as will be shown below. After irradiation, the substrates were removed from the vacuum chamber and the upper surface of the metal film was investigated using optical microscopy and atomic force microscopy (AFM).

\section{Results and discussion}

\subsection{Blister morphology}

Figure 2 shows a comparison of the blister region for single-shot back-irradiation (i.e. passing through the glass before interacting with the "back" surface of the metal film) of thin titanium films on glass microscope slides for both ns and fs laser pulses under conditions where there is efficient BB-LIFT particle removal. There is an obvious difference in the resulting topographies when studied postirradiation. In the ns case, the front surface is relatively smooth and flat whereas there is an obvious "bump" with a height of ca. 100-200 $\mathrm{nm}$ remaining on the front surface after fs irradiation.

\subsubsection{Nanosecond irradiation}

For ns laser irradiation, the blisters were formed with laser fluences ranging from 220 to $320 \mathrm{~mJ} \mathrm{~cm}^{-2}$. They tend to be flat on post-irradiation inspection with a slightly raised ring of $\sim 5 \mathrm{~nm}$ height as measured by AFM, seen at a distance of around $30 \mu \mathrm{m}$ in Fig. 2a. This is very similar to topographies observed for ns front-irradiation of metals [15]. For higher fluence, more cracks are formed on the blisters, Fig. 3, before the entire metal film is removed for fluences beyond ca. $400 \mathrm{~mJ} \mathrm{~cm}^{-2}$, for the 300 - to $380-\mathrm{nm}$ thick films studied here. Figure 4 shows the blister region, obtained when irradiating the backside of the metal film through the glass substrate, and the surface structure obtained after irradiating the front side of the metal film with ns pulses of a similar absorbed fluence. The similarity of these images indicates that the film is heated almost uniformly across its thickness on the timescale of the laser pulse. This is shown below via simulation.

We have previously studied ns BB-LIFT from Ti-coated glass slides for ejection of gold-coated silica nanoparticles

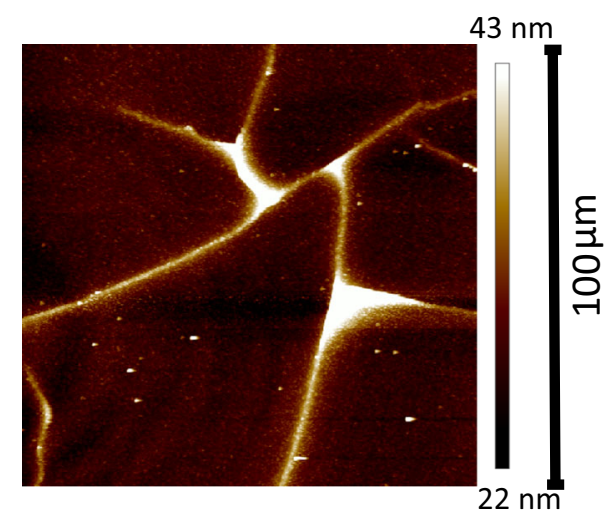

Fig. 3 AFM scan of the surface of a ns blister formed at a laser fluence of $220 \mathrm{~mJ} \mathrm{~cm}{ }^{-2}$ showing the formation of cracks on the surface. The area scanned is $100 \times 100 \mu \mathrm{m}^{2}$
Fig. 2 Blisters formed by single-shot irradiation. a ns laser pulse $220 \mathrm{~mJ} \mathrm{~cm}^{-2}$ with film thickness $380 \mathrm{~nm}$ and $\mathbf{b}$ fs laser

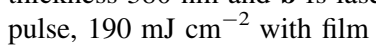
thickness $200 \mathrm{~nm}$. In each case the left hand image is an AFM scan in tapping mode showing the topography (due to the larger size of the ns blister, this is a zoomed-in region of the actual blister, an optical microscope image of the full blister can be seen in Fig. 4a). The right-hand image shows a cross section of the AFM scan at the position indicated by the straight line on the image
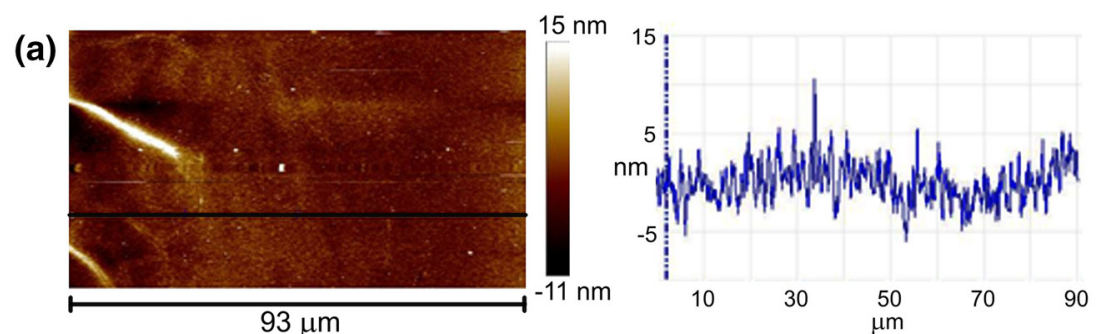

(b)
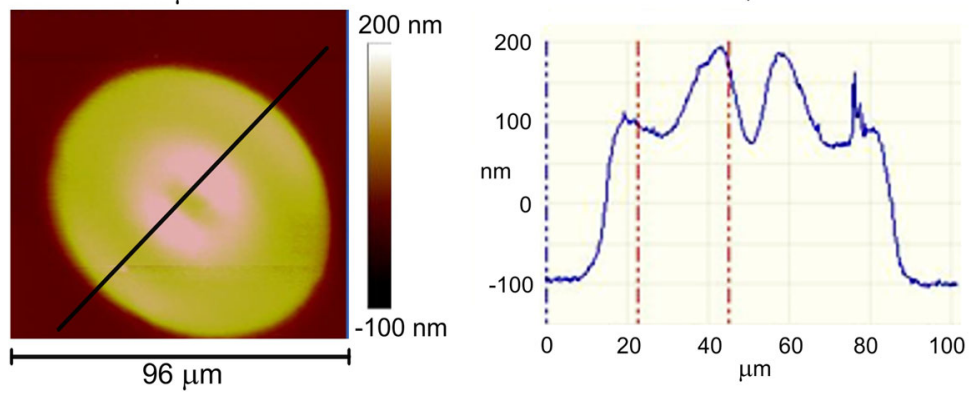


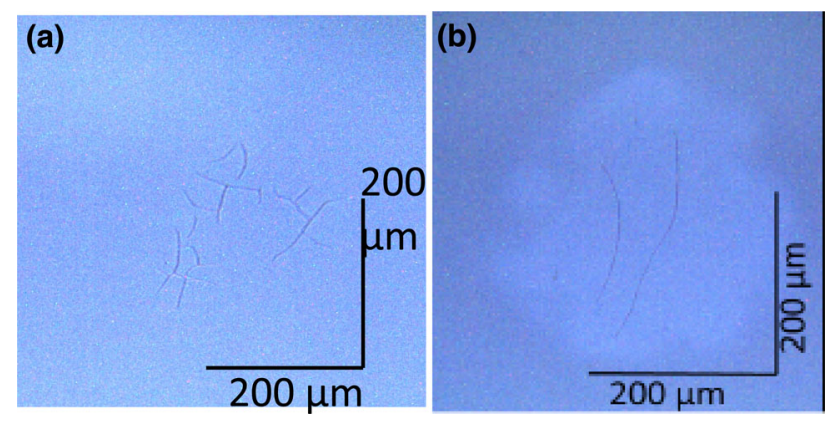

Fig. 4 Optical microscope images of the front surface of the metal films after ns pulse irradiation a irradiation through the glass substrate (back-irradiation), $220 \mathrm{~mJ} \mathrm{~cm} \mathrm{~cm}^{-2}$ and $\mathbf{b}$ direct irradiation at the front surface (front-irradiation), $315 \mathrm{~mJ} \mathrm{~cm}^{-2}$. Due to the different reflectivities, the absorbed energy is similar in both cases (135 and $160 \mathrm{~mJ} \mathrm{~cm}{ }^{-2}$, respectively) consistent with the onset of cracking in both cases. Film thickness $380 \mathrm{~nm}$

for gas phase analysis [6]. In that work, although the nanoparticle velocity distribution provided evidence of a mechanical, impulsive removal from the surface with particle velocities of ca. $50 \mathrm{~m} \mathrm{~s}^{-1}$, consistent with rapid deformation and impulsive energy transfer, the pattern of remaining particles on the surface, together with estimations of the temperature in the thin metal film, provided evidence for at least partial melting of the metal [6]. Here we have performed a more detailed theoretical analysis of Ti film heating under ns BB-LIFT conditions using a thermal model $[16,17]$. The model is based on solving the heat flow equation in its one-dimensional form that allows the evolution of the temperature profile $T(t, z)$ to be analysed along the film thickness direction $z$ :

$$
\begin{gathered}
\left(c_{\mathrm{f}} \rho_{\mathrm{f}}+L_{\mathrm{m}} \delta\left(T-T_{\mathrm{m}}\right)+L_{\mathrm{t}} \delta\left(T-T_{\mathrm{t}}\right)\right) \frac{\partial T}{\partial t} \\
=\frac{\partial}{\partial z} \lambda_{\mathrm{f}} \frac{\partial T}{\partial z}+S(t, z), \quad z>0
\end{gathered}
$$

and inside the glass substrate

$c_{\mathrm{g}} \rho \frac{\partial T}{\partial t}=\frac{\partial}{\partial z} \lambda_{\mathrm{g}} \frac{\partial T}{\partial z}, \quad z<0$

with the film-substrate interface located at $z=0$. Here $\rho, c$, and $\lambda$ are the mass density, heat capacity and thermal conductivity, respectively. The indices $f$ and $g$ refer to the film and glass, respectively. The laser pulse energy is assumed to be absorbed only in the film and thus the source term is given by $S(t, z)=(1-R) \alpha_{\mathrm{f}} I(t) \exp \left(-\alpha_{\mathrm{f}} z\right)$ at $z>0$, where $I(t)$ is the incident laser beam intensity, $R$ is the total reflection coefficient, and $\alpha_{\mathrm{f}}$ is the absorption coefficient of titanium. The terms $L_{\mathrm{m}} \delta\left(T-T_{\mathrm{m}}\right)$ and $L_{\mathrm{t}} \delta\left(T-T_{\mathrm{t}}\right)$ allow for calculations across the phase transition interfaces at the melting temperature $T_{\mathrm{m}}$ and the temperature $T_{\mathrm{t}}$ of the $\alpha-\beta$ phase transformation in titanium $[18,19]$. Here, $L_{\mathrm{m}}$ and $L_{\mathrm{t}}$ are the latent heats of fusion and the $\alpha-\beta$ transition, respectively [16]. The values used in the calculations are summarised in Table 1. Note that vaporisation of the Ti film is not included in the model since we consider relatively low metal temperatures, below or near the melting point.

Equations (1) and (2) are solved with the following initial and boundary conditions

$$
\begin{gathered}
T(0, z)=T_{0},\left.\quad \lambda_{\mathrm{f}} \frac{\partial T}{\partial z}\right|_{z=0}=\left.\lambda_{\mathrm{g}} \frac{\partial T}{\partial z}\right|_{z=0},\left.\quad \lambda_{\mathrm{f}} \frac{\partial T}{\partial z}\right|_{z=l}=0 \\
\left.\lambda_{\mathrm{g}} \frac{\partial T}{\partial z}\right|_{z=-d}=0
\end{gathered}
$$

where $T_{0}$ is the initial (room) temperature of the filmsubstrate system, $l$ and $d$ are the thicknesses of the film and substrate, respectively. In the calculations, the $d$ value is taken as $5 \mu \mathrm{m}$ to be much larger than the $l$ value $(300 \mathrm{~nm})$. It was checked that further increase in the substrate thickness to approach the experimental value did not affect the modelled metal film temperature.

Figure 5a shows the temporal evolution of the upper surface temperature $T_{\mathrm{s}}$ of the titanium film under ns BBLIFT for several laser fluences within the studied fluence range. The upper surface reaches its maximum temperature at $\sim 10 \mathrm{~ns}$ after the laser pulse peak, providing us with a timescale for heating of the film. The peak $T_{\mathrm{s}}$ value varies in the range from ca. $1400 \mathrm{~K}$ at $0.17 \mathrm{~J} \mathrm{~cm}^{-2}$ to $2040 \mathrm{~K}$ at $0.32 \mathrm{~J} \mathrm{~cm}^{-2}$. The melting point of titanium $(1941 \mathrm{~K})$ is reached at $F_{0} \approx 0.3 \mathrm{~J} \mathrm{~cm}^{-2}$ that is consistent with the estimation of Ref. [6]. Due to the low thermal conductivity of the substrate, the film remains hot for a fairly long time (e.g., at $0.25 \mathrm{~J} \mathrm{~cm}^{-2}, T_{\mathrm{s}}$ exceeds $1000 \mathrm{~K}$ during $\sim 1 \mu \mathrm{s}$ after the laser pulse, see the inset in Fig. 5a). Figure $5 \mathrm{~b}$ shows typical temperature distributions across the filmsubstrate system at approximately $10 \mathrm{~ns}$ after the laser pulse maximum. At this time, the metal film has been heated very uniformly across its thickness so that the difference in temperature between the inner and upper surfaces does not exceed $20 \mathrm{~K}$. In contrast, the temperature drops quickly within the glass substrate, reaching the initial room temperature within a distance of $200-300 \mathrm{~nm}$ (Fig. 5b).

After the laser pulse has terminated, the temperature will decrease and the film will contract again leading to the smooth and relatively flat film surface seen in Fig. 2a. The onset of cracks in the surface can be attributed to thermal stress that the film experiences when heated. Again, following the arguments detailed in [6], we can estimate the thermal stress, $\sigma$, using the expression

$\sigma=\frac{E k_{\mathrm{e}} \Delta T}{2(1-v)}$

where $E$ is the Young's modulus of the film (103-110 GPa, the lower value corresponds to the lower limit of thin film 
Table 1 Properties of materials used in the calculations for ns irradiation at $532 \mathrm{~nm}$

\begin{tabular}{|c|c|c|}
\hline Parameter & Titanium & Glass \\
\hline$\rho\left(\mathrm{g} \mathrm{cm}^{-3}\right)$ & $\begin{array}{l}4.55-0.000147 T, T<1941 \mathrm{~K}[20] \\
4.12, T>1941 \mathrm{~K}[20]\end{array}$ & $2.2 *$ \\
\hline$c\left(\mathrm{~J} \mathrm{~kg}^{-1} \mathrm{~K}^{-1}\right)$ & $\begin{array}{l}488+0.00293 T+0.00048 T^{2}-3.55 \mathrm{e}-7 T^{3}, 300<T<1200 \mathrm{~K}[20] \\
2055-2.94 T+0.0019 T^{2}-3.63 \mathrm{e}-7 T^{3}, 1200<T<1941 \mathrm{~K}[20] \\
\text { 989.2. } T>1941 \mathrm{~K}[20]\end{array}$ & $800[21]$ \\
\hline$\lambda\left(\mathrm{Wm}^{-1} \mathrm{~K}^{-1}\right)$ & $\begin{array}{l}-0.013(T-300)+22.2,300<T<500 \mathrm{~K}[20] \\
-0.00053(T-500)+19.6,500<T<1156 \mathrm{~K}[20] \\
0.01693(T-1156)+19.95, T>1156 \mathrm{~K}[20]\end{array}$ & $0.8[21]$ \\
\hline$\alpha_{\mathrm{f}}\left(\mathrm{cm}^{-1}\right)$ & $6 \times 10^{5}[22]$ & - \\
\hline$T_{\mathrm{m}}(\mathrm{K})$ & $1941[14]$ & - \\
\hline$L_{\mathrm{m}}\left(\mathrm{kJ} \mathrm{mol}^{-1}\right)$ & $14.15[14]$ & - \\
\hline$T_{\mathrm{t}}(\mathrm{K})$ & $1250[18]$ & - \\
\hline$L_{\mathrm{t}}\left(\mathrm{kJ} \mathrm{mol}^{-1}\right)$ & 4.3 [19] & - \\
\hline$R$ & $0.36^{*}$ & $0.04 *$ \\
\hline
\end{tabular}

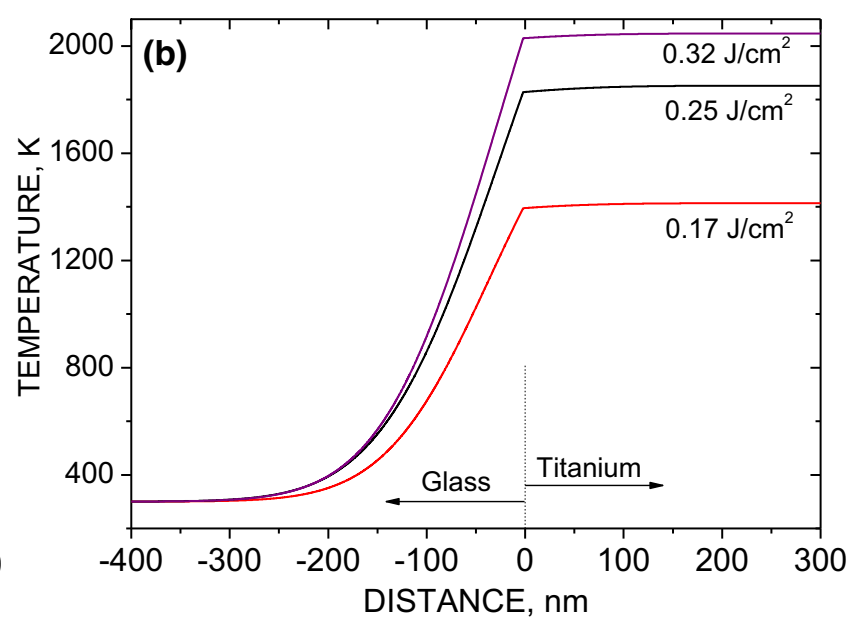

shown by the dashed line. b Temperature distributions through the film and substrate at the times when the maximum temperature of the film surface is reached

that described the BB-LIFT of nanoparticles from a thin Ti film in terms of a thermal stress mechanism [6].

\subsubsection{Femtosecond irradiation}

The morphology of the blisters produced by the fs laser pulses, once the substrate has cooled, is quite different as can be seen clearly in Fig. 2. They do not collapse back to the height of the original metal film but remain protruded from the substrate. The dimension of the protruded blister in the fs case is significantly smaller than the size of the laser spot (ca. $100 \mu \mathrm{m}$ diameter for a $300 \mu \mathrm{m}$ diameter laser spot), in contrast to the area of the surface affected by the ns pulse irradiation that closely matches the laser spot 
size. Due to the much smaller heat-affected zone for irradiation with fs pulses, the metal film is not uniformly heated throughout its thickness during the laser irradiation.

The different morphologies and their dependence on laser fluence can be explained by invoking different mechanisms for the deformation of the metal films. Thermal expansion, as discussed above, can explain the observations using ns laser pulses. For fs laser pulses, an explanation in terms of metal film ablation at the interface with the glass substrate is more appropriate. The absorbed laser energy is concentrated within a narrow layer adjacent to the film-substrate interface with a thickness corresponding to the irradiation penetration depth, $\alpha$, in titanium. For $800 \mathrm{~nm}, \alpha=800 / 4 \pi k=19 \mathrm{~nm}$, where $k=3.33$ is the extinction index of $\mathrm{Ti}$ [22]. For instance, for the example shown in Fig. 2b, the absorbed energy density is ca. $100 \mathrm{~kJ} \mathrm{~cm}^{-3}$ that is significantly larger than the $50 \mathrm{~kJ} \mathrm{~cm}^{-3}$ threshold energy for ablation by 800-nm fs laser pulses, determined by Zhu et al. [25]. The absorption will result in the formation of a vapour that will rapidly expand, pressing the remaining metal film upwards and forming the blister.

The assumption of a small heat-affected zone is supported by estimating the heat transfer in the thin film under the fs irradiation conditions. For fs irradiation regimes, the electron subsystem absorbs light during the laser pulse, while the lattice stays cold. In such a highly non-equilibrium regime, the electrons provide heat transfer across the film. The electron thermal conductivity is a strong function of the electron temperature, $T_{\mathrm{e}}[26,27]$ and can be expressed as

$\lambda_{\mathrm{e}}=\frac{v_{\mathrm{F}}^{2} c_{\mathrm{e}}\left(T_{\mathrm{e}}\right)}{3\left(A T_{\mathrm{e}}^{2}+B T_{1}\right)}$

where $v_{\mathrm{F}}$ is the Fermi velocity, $c_{\mathrm{e}}$ is the heat capacity of the electronic subsystem, $T_{1}$ is the lattice temperature, $A=6.16 \times 10^{4} \mathrm{~K}^{-2} \mathrm{~s}^{-1}$ and $B=1.21 \times 10^{13} \mathrm{~K}^{-1} \mathrm{~s}^{-1}$ [27]. From the heat transfer equation for the electron subsystem, the heat propagation distance as a function of time $\Delta t$ can be estimated as $\Delta x \sim \sqrt{\chi_{\mathrm{e}} \Delta t}$ where $\chi_{\mathrm{e}}$ is the thermal diffusivity. The latter can be estimated as

$\chi_{\mathrm{e}} \sim \frac{\lambda_{\mathrm{e}}}{c_{\mathrm{e}}}=\frac{v_{\mathrm{F}}^{2}}{3\left(A T_{\mathrm{e}}^{2}+B T_{1}\right)}$

with $v_{\mathrm{F}}=3 \times 10^{5} \mathrm{~m} \mathrm{~s}^{-1}$ [28].

The electron-lattice thermalisation in metals normally takes place on timescales ranging from several ps to several dozens of ps [29]. However, titanium has an extremely high electron-lattice coupling factor, higher than $10^{18} \mathrm{Wm}^{-3} \mathrm{~K}^{-1}$ [26] and the thermalisation process has occurred already during the first picosecond [27].

For conditions similar to those used in the experiments, with a laser fluence of $200 \mathrm{~mJ} \mathrm{~cm}^{-2}$, the electron temperature at the interface between the metal and glass substrate is estimated to be $32,000 \mathrm{~K}$ after a time of $1 \mathrm{ps}$ after the termination of the laser pulse. At such high electron temperatures, the thermal diffusivity of the electrons, $\chi_{\mathrm{e}}$ (Eq. 6), is still slightly dependent on the electron temperature, but due to the very efficient electron-lattice thermalisation and lattice heating, titanium will be ablated at the film/substrate interface. Metal ablation onsets after ca. 10 ps [30]. By this time the heat-affected zone in the $\mathrm{Ti}$ film will have increased by only $\Delta x \sim \sqrt{\chi_{\mathrm{e}} \Delta t} \sim 16 \mathrm{~nm}$ and is still much smaller than the film thickness. At the same time, the lattice temperature at the interface will exceed $10,000 \mathrm{~K}$, leading to intensive ablation with a species velocity of several $\mathrm{km} \mathrm{s}^{-1}$ [30], thus creating a strong recoil force on the film.

An important conclusion can be made concerning the mechanism of blister formation in fs irradiation regimes. For relatively thick metal films, as in our case, when the metal film is heated to a depth that is much smaller than the film thickness, the blister formation will occur predominantly via metal ablation at the film-substrate interface. For films where the thickness is of the order or smaller than the heat propagation distance, by the end of the electron-lattice thermalisation process, blister formation should proceed via the radial stress in the irradiation spot region for films quasi-uniformly heated throughout their thickness, as we observe for the ns irradiation case. For intermediate regimes, the two mechanisms can be expected to coexist.

\subsection{Multiple shots}

Further evidence for the two different blister formation mechanisms can be found in Fig. 6 where the influence of multiple laser shots on one position is shown for both fs pulses (Fig. 6a-c) and ns pulses (Fig. 6d-f).

The surface structure after ns pulse irradiation does not show any significant change in diameter or topography for multiple shots. The main difference for the conditions used to obtain the blisters in Fig. 6 is that there may be a slight increase in the density of cracks as the number of laser shots is increased. In contrast, with the fs pulses there are clear differences from shot to shot. The affected area increases with rings visible to mark the region influenced by the previous laser shots. Under the fluence conditions, near the ablation threshold, used to obtain the images in Fig. 6a, the blister remains intact after the first laser shot. However, a large visible crack appears on the blister after the second laser shot and the film has been disrupted and lifted off the substrate for the seventh shot. The combination of ablation of a thin layer and expansion of the film will contribute to the thinning of the metal film, as each pulse ablates a little more of the metal at the glass/metal interface and the film re-expands on each laser shot until 
Fig. 6 Optical microscope images of the blisters formed by fs (top) and ns (bottom) laser pulses. Top fs laser fluence of $210 \mathrm{~mJ} \mathrm{~cm}^{-2}, 310 \mathrm{~nm}$ thick film a single shot, $\mathbf{b}$ double shot, c seven shots. Bottom ns laser fluence of $270 \mathrm{~mJ} \mathrm{~cm} \mathrm{~cm}^{-2}, 380$ nm thick film d single shot, e double shot, $\mathbf{f}$ ten shots
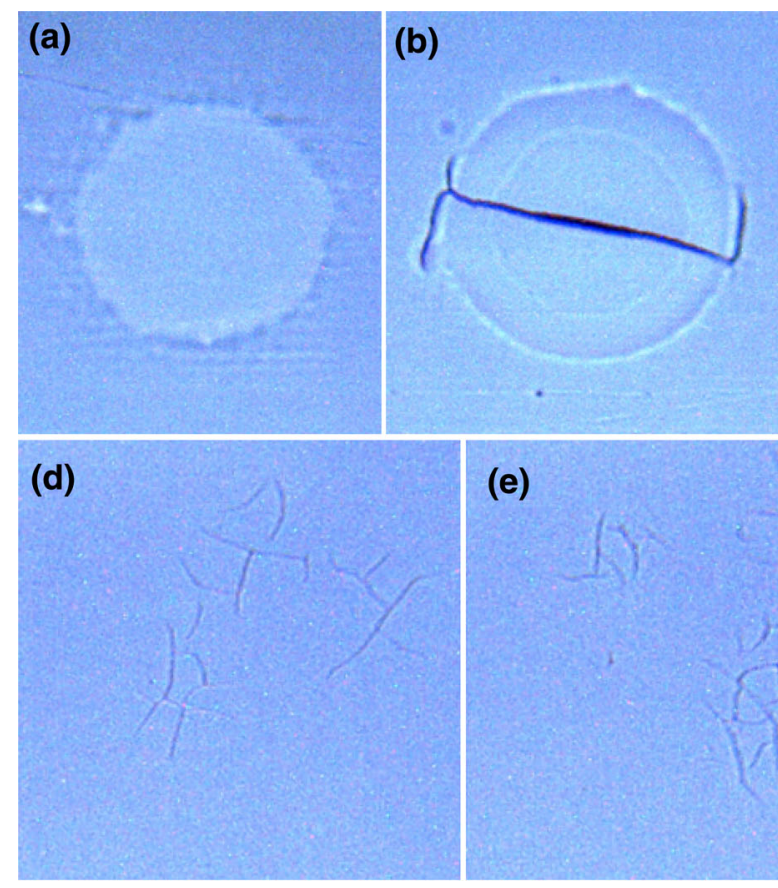

(e)

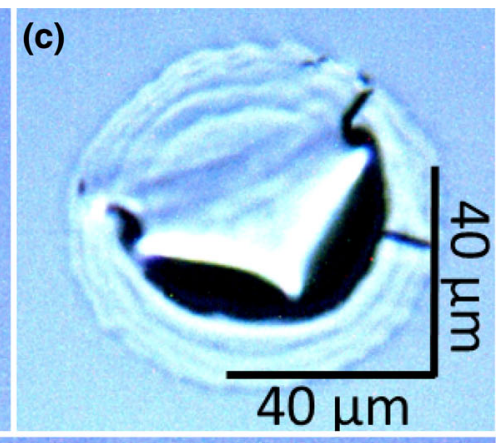

(f) (a)

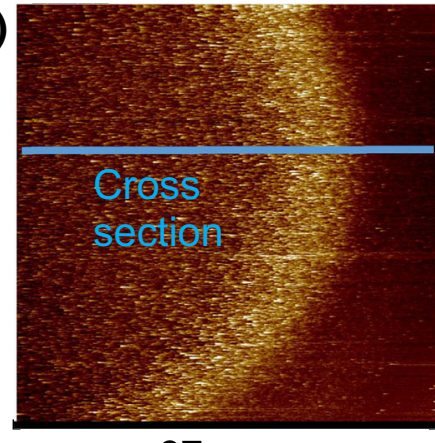

$67 \mu \mathrm{m}$
Fig. 7 Topography of the top surface of a $310 \mathrm{~nm}$ thick Ti a fs laser pulse of fluence $200 \mathrm{~mJ} \mathrm{~cm}^{-2}$

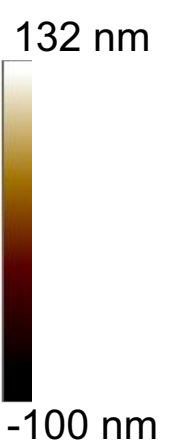

$-100 \mathrm{~nm}$

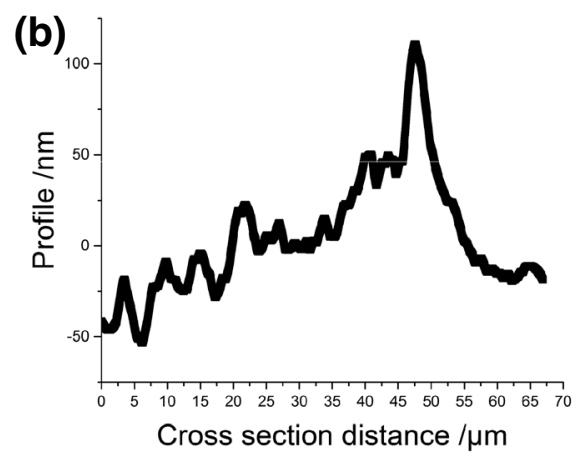

Cross section distance $/ \mu \mathrm{m}$

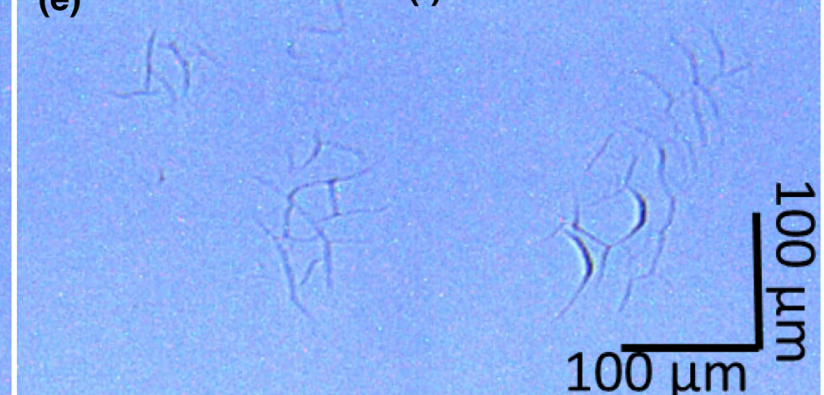

the film ruptures. The induced pressure exceeds the elastic limit, and the film does not relax back to its starting position, unlike the situation with thermal expansion and contraction observed for the ns pulses. Some rippling can be observed on top of the raised area in Fig. $2 b$ that may be a consequence of inward pressure on the film after the ablated vapour has cooled, decreasing the pressure under the blister "dome".

To check that the fs laser irradiation conditions exceed the ablation threshold, we compare the top surface of the $\mathrm{Ti}$ film after direct radiation with a fs pulse. Figure 7 illustrates the result for a laser fluence of ca. $200 \mathrm{~mJ} \mathrm{~cm}^{-2}$ [corresponding to an absorbed fluence of $90 \mathrm{~mJ} \mathrm{~cm}^{-2}$ $(R=0.55[22])]$, similar to the absorbed fluence for the conditions of Fig. $2 \mathrm{~b}$ with $R=0.45$. There is clear evidence for ablation having taken place with removal of material from the irradiation spot centre and a raised edge due, presumably, to a shock wave produced by the ablation process.

\subsection{AFM force-distance measurements}

Further insight and confirmation of the different blister formation mechanisms can be obtained from AFM forcedistance measurements, where the force needed to deflect the material is determined. Figure 8a shows an AFM image of a fs-laser-induced blister used for the force-distance measurements given in Fig. 8b. There is a clear difference in the measurements depending on whether the AFM tip is situated at the centre of the raised blister or off-blister. The difference in the distances for the onset of the tip deflection corresponds to the height of the centre of the blister. Less force is needed to deflect the film, at the centre of the blister "dome", consistent with the film being raised from 
Fig. 8 AFM images and forcedistance curves. a, b fs laser $185 \mathrm{~mJ} \mathrm{~cm}^{-2}, 310 \mathrm{~nm}$ thick film. Black dots in (a) indicate positions for force-distance measurements in (b). c, $\mathbf{d} \mathrm{ns}$ laser $270 \mathrm{~mJ} \mathrm{~cm}{ }^{-2}, 380 \mathrm{~nm}$ thick film. Bright dots in (c) indicate positions for forcedistance measurements in (d) (a)

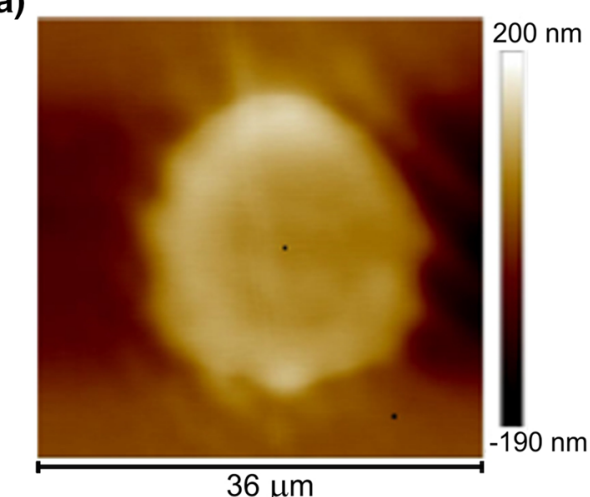

(c)

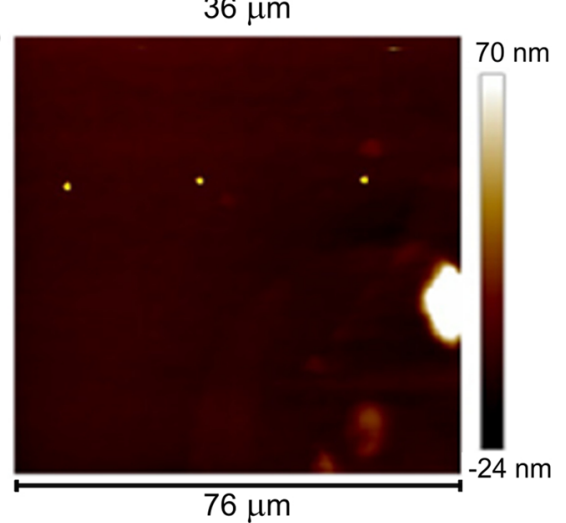

(b)

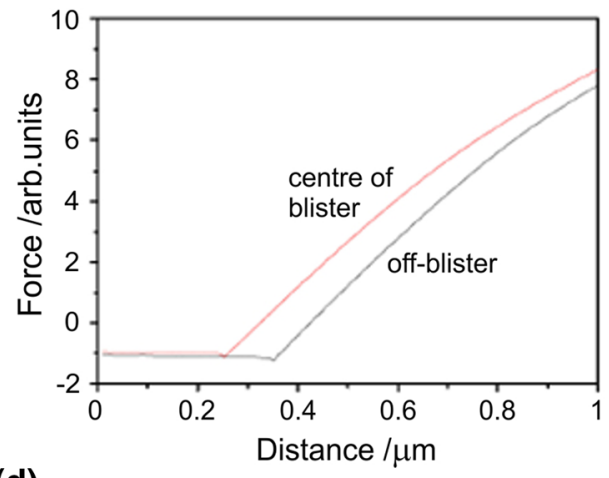

(d)

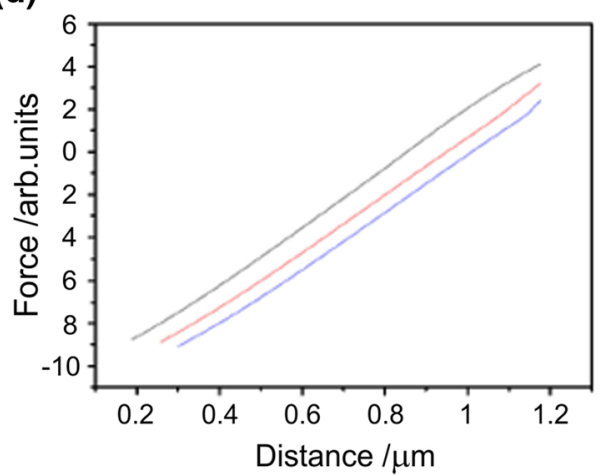

the underlying glass substrate. Similar measurements are shown for the ns blister in Fig. 8c, d. Here, there is no obvious difference in the slope of the plots, indicating that the film remains in contact with the underlying glass substrate across the blister region.

\section{Conclusions}

Optical microscopy and AFM have been used to study the morphology and mechanical properties of blisters produced in thin Ti films deposited on glass. Laser-induced blister formation has been shown previously to provide a convenient way of releasing nanoparticles or large molecules into the gas phase as well as for microscale patterning via direct transfer of deposited species to a target surface [11]. Two mechanisms have been discussed in this context; a thermomechanical mechanism based on induced thermal stress and an ablative mechanism in which a thin layer of the metal film is vaporised at the interface with the glass substrate $[7,8]$. In the present study, we show that both mechanisms can be clearly distinguished by studying the metal film surfaces after blister formation. Nanosecond laser pulses lead to heating of the metal film quasi-uniformly across its thickness. The induced thermal stress then leads to deformation that can inject particles into the gas phase. The hot film will then relax back to its initial position on cooling. For relatively high laser fluences, the thermal stress will exceed the yield stress for a thin Ti film and cracks will appear on the surface. For fs laser pulses, the laser pulse induces ablation of the metal film at the interface with the glass substrate, producing a rapid gas expansion that can mechanically deform the film, providing the mechanism for particle ejection. In this case, the expanded film does not return to its initial position on cooling but a well-defined blister remains on the surface. These two quite different mechanisms can have consequences for the application of BB-LIFT for the fragmentation-free ejection of large molecules into the gas phase. In the ns case, a significant fraction of the transferred particles can be strongly heated.

Acknowledgments Financial support from the EU via Marie Curie International Incoming Fellowship Grant No. 302991 and from the Leverhulme Trust (RPG 248) is gratefully acknowledged. SVS, NMB and AVB acknowledge support from the Russian Foundation for Basic Research (Project No. 15-08-06591). NMB acknowledges support of the state budget of the Czech Republic (project HiLASE: Superlasers for the real world: LO1602). EEBC acknowledges the hospitality and financial support from JILA in the form of a Visiting Fellowship.

Open Access This article is distributed under the terms of the Creative Commons Attribution 4.0 International License (http://crea tivecommons.org/licenses/by/4.0/), which permits unrestricted use, distribution, and reproduction in any medium, provided you give appropriate credit to the original author(s) and the source, provide a link to the Creative Commons license, and indicate if changes were made. 


\section{References}

1. A.M. Bustos, J. Encinar, A. Sanz-Medel, Mass spectrometry for the characterisation of nanoparticles. Anal. Bioanal. Chem. 405, 5637-5643 (2013)

2. A. Stolow, Femtosecond time-resolved photoelectron spectroscopy of polyatomic molecules. Annu. Rev. Phys. Chem. 54, 89-119 (2003)

3. P. Biswas, C.-Y. Wu, Nanoparticles and the environment. J. Air Waste Manag. Assoc. 55, 708-746 (2005)

4. M. Arndt, De Broglie's meter stick: making measurements with matter waves. Phys. Today 67, 30-36 (2014)

5. A.R. Dow, A.M. Wittig, H.I. Kenttämaa, Laser induced acoustic desorption mass spectrometry. Eur. J. Mass Spectrom. 18, 77-92 (2012)

6. A.V. Bulgakov, N. Goodfriend, O. Nerushev, N.M. Bulgakova, S.V. Starinskiy, Y.G. Shukhov, E.E.B. Campbell, Laser-induced transfer of nanoparticles for gas-phase analysis. J. Opt. Soc. Am. B 31, C15-C21 (2014)

7. T.V. Kononenko, P. Alloncle, V.I. Konov, M. Sentis, Laser transfer of diamond nanopowder induced by metal film blistering. Appl. Phys. A 94, 531-536 (2009)

8. M.S. Brown, N.T. Kattamis, C.B. Arnold, Time-resolved study of polyimide absorption layers for blister-actuated laser-induced forward transfer. J. Appl. Phys. 107, 083103 (2010)

9. N.T. Kattamis, N.D. McDaniel, S. Bernhard, C.B. Arnold, Laser direct write printing of sensitive and robust light emitting organic molecules. Appl. Phys. Lett. 94, 103306 (2009)

10. T.V. Kononenko, I.A. Nagovitsyn, G.K. Chudinova, I.N. Mihailescu, Clean, cold, and liquid-free laser transfer of biomaterials. Laser Phys. 21, 823-829 (2011)

11. N.T. Kattamis, N.D. McDaniel, S. Bernhard, C.B. Arnold, Ambient laser direct-write printing of a patterned organo-metallic electroluminescent device. Org. Electron. 12, 1152-1158 (2011)

12. T.V. Kononenko, I.A. Nagovitsyn, G.K. Chudinova, I.N. Mihailescu, Application of clean laser transfer for porphyrin micropatterning. Appl. Surf. Sci. 256, 2803-2808 (2010)

13. W. Fang, C.-Y. Lo, On the thermal expansion coefficients of thin films. Sens. Actuators A 84, 310-314 (2000)

14. W.M. Haynes (ed.), Handbook of Chemistry and Physics, 96th edn. (CRC Press, Boca Raton, 2015)

15. D.W. Bäuerle, Laser Processing and Chemistry, 4 edn. (Springer, Berlin Heidelberg, 2011), p. 851. http://www.springer.com/us/ book/9783642176128. Accessed 6 Nov 2015
16. N.M. Bulgakova, A.V. Bulgakov, L.P. Babich, Energy balance of pulsed laser ablation: thermal model revised. Appl. Phys. A 79, 1323-1326 (2004)

17. N.M. Bulgakova, A.B. Evtushenko, Y.G. Shukhov, S.I. Kudryashov, A.V. Bulgakov, Role of laser-induced plasma in ultradeep drilling of materials by nanosecond laser pulses. Appl. Surf. Sci. 257, 10876-10882 (2011)

18. M.M. Martynyuk, V.I. Tsapkov, Electrical resistance, enthalpy and phase transitions of titanium, zirconium and hafnium during pulsed heating. Russ. Metall. (Metally) 2, 181-188 (1974)

19. E. Kaschitz, P. Reiter, Enthalpy and temperature of the titanium alpa-beta phase transition. Int. J. Thermophys. 23, 1339-1345 (2002)

20. V.E Zinoviev, Thermophysical Properties of Materials at High Temperatures. Reference Edition-M, Metallurgiya (Rus.) (1989)

21. http://www.saint-gobain-sekurit.com/glossary/glass-properties. Accessed 17 Dec 2015

22. E.D. Pallik, Handbook of Optical Constants of Solids (Academic Press, Cambridge, 2008)

23. M. Chinmulgund, R.B. Inturi, J.A. Barnard, Effect of Ar gas pressure on growth, structure, and mechanical properties of sputtered $\mathrm{Ti}, \mathrm{Al}$, Tial, and Ti3al films. Thin Solid Films 270, 260-263 (1995)

24. Z. Shan, S.K. Sitaraman, Elastic-plastic characterization of thin films using nanoindentation technique. Thin Solid Films 437, 176-181 (2003)

25. X. Zhu, D.M. Villeneuve, A.Y. Naumov, S. Nikumb, P.B. Corkum, Experimental study of drilling sub-10 Mm holes in thin metal foils with femtosecond laser pulses. Appl. Surf. Sci. 152, 138-148 (1999)

26. Z. Lin, L.V. Zhigilei, V. Celli, Electron-phonon coupling and electron heat capacity of metals under conditions of strong electron-phonon nonequilibrium. Phys. Rev. B 77, 075133 (2008)

27. Y. Levy, T.J.Y. Derrien, N.M. Bulgakova, E.L. Gurevich, T. Mocek, Relaxation dynamics of femtosecond-laser-induced temperature modulation on the surfaces of metals and semiconductors. Appl. Surf. Sci. (2016). doi:10.1016/j.apsusc.2015.10.159

28. P.B. Allen, in Quantum Theory of Real Materials, ed. by J.R. Chelikowsky, S.G. Louie (Kluwer, Boston, 1996), p. 219

29. S.S. Wellershoff, J. Hohlfeld, J. Güdde, E. Matthias, The role of electron-phonon coupling in femtosecond laser damage of metals. Appl. Phys. A 69, S99-S107 (1999)

30. C. Wu, L. Zhigilei, Microscopic mechanisms of laser spallation and ablation of metal targets from large-scale molecular dynamics simulations. Appl. Phys. A 114, 11-32 (2014) 